\title{
A Framework for Intersection Traffic Safety Screening with the Implementation of Complex Network Theory
}

\author{
Xueyu Mi, ${ }^{1,2}$ Chunfu Shao, ${ }^{1}$ Chunjiao Dong $\mathbb{D}^{1}{ }^{1}$ Chengxiang Zhuge, ${ }^{3}$ and Yan Zheng ${ }^{1}$ \\ ${ }^{1}$ Key Laboratory of Transport Industry of Big Data Application Technologies for Comprehensive Transport, Ministry of Transport, \\ Beijing Jiaotong University, Beijing 100044, China \\ ${ }^{2}$ College of Civil and Architectural Engineering, North China University of Science and Technology, He Bei, \\ Tang Shan 063210, China \\ ${ }^{3}$ Department of Land Surveying and Geo-Informatics, The Hong Kong Polytechnic University, Hong Kong, China
}

Correspondence should be addressed to Chunjiao Dong; cjdong@bjtu.edu.cn

Received 12 August 2020; Revised 7 September 2020; Accepted 13 September 2020; Published 27 September 2020

Academic Editor: Feng Chen

Copyright (c) 2020 Xueyu Mi et al. This is an open access article distributed under the Creative Commons Attribution License, which permits unrestricted use, distribution, and reproduction in any medium, provided the original work is properly cited.

The traffic safety screening could provide guidance for determining the use of resources for traffic safety improvements and is critical for the traffic management. To account for the impacts of traffic safety and the effects of intersection as a node in the roadway network, a framework with six indicators and four strategies is proposed for intersection traffic safety screening. The traffic flow has been incorporated into the process of developing three indicators from the perspective of the complex network theory to measure the node importance. For the assessment of traffic safety, other than the observed and estimated crash frequency and modified time-to-collision, a distance that describes the nonlane-based movements has been proposed from the perspective of traffic safety. A multilayer entropy-weighted VIKOR (MEW-VIKOR) approach is proposed to compute the ranking results, and four strategies have been developed to better account for the effects of the six indicators simultaneously. A roadway network with 28 intersections in Shenzhen has been adopted to verify the effectiveness of the proposed framework for intersection traffic safety screening. The results indicate that the proposed framework with two layers could represent the features of traffic safety and the characteristics of node importance and satisfy the expectation from the public, government, and research institutes. With an appropriate threshold setting, the ranking results are consistent with the intersection safety investigation and contribute significantly to the reduction of false-positive and false-negative cases in identifying the black spot intersections.

\section{Introduction}

Intersections indicate a complicated and hazardous roadway environment involving human factors, vehicle characteristics, roadway design features, and traffic flow movements. The presence of conflicted traffic flow movements, mixed roadway users, diversified driver actions and behaviors, and complicated roadway design features creates a challenging and confusing condition to the roadway users that leads to greater crash frequencies with serious severity. Traffic crashes at intersections place a huge burden on society and public in terms of death, injury, and property damage $[1,2]$. Once a traffic crash occurred at an intersection, as the critical component and important node of the roadway network, the condition of the intersection will impact the operation efficiency and safety of neighboring zones, and even the overall roadway network. The influencing degree and extent depend on the importance of the intersection as a node in the roadway network. Hence, the measurement of importance of intersection as a node in the roadway networks can provide insights into understanding the spread rate and extent of traffic congestions and the loss of network efficiency that caused by the occurrences of traffic crashes. To reduce the impacts of intersection-related traffic crashes on the roadway network, there is a need to identify the most important intersections with traffic safety issues in the roadway network and ensure that the necessary countermeasures are designed and applied to prevent the occurrences of traffic crashes and reduce the crash severity. 


\section{Literature Review}

As part of the roadway management process, the traffic safety screening of hazardous intersections and identification of high-risk traffic crash intersections are of great interest from safety specialists and roadway agencies. In the process of traffic safety analyses, the term of black spot (BS) is adopted to describe the locations that have a greater crash rates than an average. The identification, ranking, and treatment of black spots have been referred to a standard methodology of safety management for over 30 years, which effectively reduce and prevent the occurrences of traffic crashes [3]. Since conducting a detailed engineering study for all roadway locations is expensive, the traffic safety screening is critical, which helps the engineers to identify the locations with potential for safety improvements. For the roadway network screening, the most crash-prone sites can be identified. The identified locations can be the school zones, corridors, intersections, and others. In practice, screening at intersection level for the entire roadway network is required for various reasons that include the need to meet drivers' expectations of homogeneous infrastructure conditions across the roadway network.

Athough the methods, such as the Empirical Bayes (EB) method, are recommended for network screening by the Highway Safety Manual (HSM), the simplistic approaches that rely only on observed crash frequency or crash severity are commonly used by researchers $[4,5]$. Rahman et al. [6] identified the most crash-prone school zones based on crash rates in Orange and Seminole Counties in Florida. Three countermeasures were proposed for the identified school zones, and the simulation experiments were designed to evaluate the implemented effects using microsimulation. Meuleners et al. [7] assessed the effectiveness of the black spot programs in Western Australia. The crash rate reduction and the economic benefits of the treatments at the treated locations were computed. The results showed that the programs have reduced the reported crash rate by $15 \%$. The estimated cost savings and net savings were 50.8 and 40.4 million Australian dollars, respectively. The benefit cost ratio across all treated locations was 4.9 .

The commonly used black spot identification (BSID) methods include crash frequency method (crashes per year or crashes per $\mathrm{km}$ per year), crash rate method (crashes per vehicle per kilometers or per vehicles), and a combination of the two methods $[8,9]$. Since the methods are not based on the statistical models, the results can vary in the analyzed period. To overcome the limitations of the commonly used BSID methods, the empirical Bayesian (EB) techniques have been proposed, which combines the features and benefits of the observed and predicted crash frequencies $[10,11]$. The crash frequencies can be predicted by using a safety performance function (SPF) that is developed from historical crash data. Based on the reliability level of the predicted crash frequencies, a statistical model could be used to weight the observed and predicted crash frequencies in the EB methods. The safety effects of a black spot program that was implemented in Flanders-Belgium have been evaluated by Pauw et al. [12] using an empirical Bayes method before and after study. The effects of general trends and the stochastic nature of crashes, including regression to the mean, have been considered. The results show that the implementation of the program has resulted in a $24-27 \%$ and $46-57 \%$ reduction in injury and fatal crashes, respectively.

Using observed crash frequencies and rates might result in a volume bias, a segment length bias, and a regression-tothe-mean bias [13]. Errors in BSID can result in falsepositive and false-negative cases [14]. In other words, crashes can occur in both safe and unsafe locations, and the essential is to identify the most dangerous locations with greater impacts. Other than the crash frequency/rate methods and the EB methods, the research explored a variety of methods in traffic safety screening. Fan et al. [15] proposed a featurebased depth neural network identifying the black spots, and the accuracy is $89 \%$. Cafiso and Di Silvestro [16] investigated the performance of safety indicators in black spot identification for two-lane rural roads. A Monte Carlo simulation was proposed to produce theoretical crash data that were used to define a priori hazardous sites. The results showed that the indicators that are based on the EB estimation should be used for safety improvement from the perspective of practice. Geurts et al. [17] conducted a sensitivity analysis regarding the identification and ranking of the black spots. The results indicated that the injury weighting values that are relating to the attitude of the traffic safety problem and the usage of estimated crash counts have important impacts on the selection and ranking of black spots in terms of ranking order and traffic safety decisions.

Though there are a large number of studies focusing on the development of BSID methods, few research studies have been conducted to analyze and compare the model performances. A site and method consistency test and total rank differences test were proposed by Cheng and Washington [11] to compare the performances of four commonly used BSID methods. The EB methods showed the best consistency among the other BSID methods and were recommended to be used as the standard in the identification of BS. The same conclusion was obtained by Montella [18], whose study also proved the effectiveness of the EB method compared with the other seven BSID methods. To understand the impacts of roadway network segmentation on the performances of BSID methods, four commonly used BSID methods (empirical Bayesian (EB), excess EB, crash frequency, and crash ratio) and four segmentation methods (spatial clustering, constant traffic volume, constant length, and the standard Highway Safety Manual segmentation method) have been analyzed by Ghadi and Török [19]. The results showed that there is a significant relationship between the performances of BSID methods and segmentation methods. In general, the EB methods have superior performance compared with other methods, regardless of the segmentation approaches. The results are consistent with that of Montella's study [18], which showed that the EB method is the most reliable method for identifying the black spots.

To identify the intersection with potential for safety improvements and understand the impacts of intersectionrelated crashes on roadway networks, in the research, a framework of intersection traffic safety screening is 
developed by considering the indicators of traffic safety and node importance. To account for the traffic safety assessment and node importance simultaneously, with the developed six indicators, four strategies are proposed in the framework of intersection traffic safety screening. One is incorporating the ranking results of node importance in the procedure of traffic safety assessment. Second is incorporating the traffic safety assessment results as a factor to calculate the node importance. The third is to compute ranking results with the developed six indicators. The fourth is combining the ranking results of traffic safety assessment and node importance. To overcome the limitation of the conventional evaluation methods that are using the subjective weigh factors, a multilayer entropy-weighted VIKOR (MEWVIKOR) method is proposed to compute the ranking results. The developed framework of intersection traffic safety screening can be used for assessing the entire intersections in the roadway network and identifying the intersections with potential for safety improvements from a combined perspective by accounting for traffic safety and node importance simultaneously. The identified intersections with a ranking order are recommended for further investigation with detailed traffic safety countermeasures.

\section{Modeling Framework and Formulation}

The intersection traffic safety screening can be defined as the process of searching and ranking the intersections in roadway networks with a greater safety risk and potential of significant impacts on the traffic flow states of the roadway network. In the research, a framework that incorporates the traffic safety assessment and node importance has been proposed for intersection traffic safety screening and a multilayer entropy-weighed VIKOR (MEW-VIKOR) method is developed for the ranking. The motivation of the research is to measure the traffic safety and node importance simultaneously in the process of traffic safety screening. It would be great that all the impact factors have been considered and more indicators have been developed. However, more indicators means more complicated interaction between the variables. In the research, the six indicators are recommended to capture the characteristics of traffic safety and node importance simultaneously. In practice, based on the data and other actual situations, the indicators can be modified under the framework of traffic safety screening.

3.1. Indicators of Intersection Node Importance. The commonly used methods of node importance can be classified into the social network-based models and system sciencebased models [20]. Based on the assumption that a node importance is relevant to the connection between the nodes, the social network models were proposed to determine the network attributes, such as shortest path, degree, and closeness to highlight the difference among nodes. With the assumption that the nodes can be removed from the network, the system science methods were proposed to determine the node importance by changing the network topology and assessing the destructiveness. In the research, the node importance of intersection is computed based on the principle of the social network-based models since the assumption of the social network-based models is acceptable and satisfied in the roadway network with the intersection as the nodes.

Given that the roadway network can be considered as an undirected network, let $\mathbf{G}=(\mathbf{I}, \mathbf{S}, \mathbf{A})$ represents the roadway network that takes the intersections as the nodes, where $\mathrm{I}=\left\{i_{1}\right.$, $\left.i_{2}, \cdots, i_{n}\right\}$ denotes the set of all intersections, $|\mathbf{I}|=n ; \mathbf{S}=\left\{s_{1}, s_{2}, \cdots\right.$, $\left.s_{l}\right\} \subseteq \mathrm{I} \times \mathrm{I}$ denotes the set of connection segments, and $|\mathbf{S}|=l$; $\mathbf{A}=\left\{a_{i j}\right\}$ denotes the adjacency matrix. Accordingly, three indicators can be defined to measure the node importance of the intersections. One is the closeness centrality [20,21], one is the betweenness centrality [22], and the third is the degree centrality. Different from the conventional closeness centrality that are measured by the path length to other nodes, the closeness centrality of intersection $i$ is computed by the sum of traffic flow and the reciprocal of the sum of path length to other intersections, which is defined as

$$
\mathrm{CC}(i)=\frac{\sum_{j=1}^{n} q_{i j}}{(1 /(n-1)) \sum_{j=1}^{n} d_{i j} q_{i j}},
$$

where CC $(i)$ is the closeness centrality of intersection $i ; q_{i j}$ is the traffic flow on the shortest path between intersections $i$ and $j$; and $d_{i j}$ denotes the length of shortest path between intersections $i$ and $j$. When there is no available path between intersections $i$ and $j$, then $d_{i j}=\infty \quad\left(1 / d_{i j}=0\right)$. The greater CC $(i)$ indicates that the $i$ intersection is more important.

The indicator of betweenness centrality indicates the importance of an intersection controlling the traffic flow along the shortest path in the roadway network, which can be defined as

$$
\mathrm{BC}(i)=\frac{2\left(\sum_{r, s \neq i}\left(t_{r s}(i) q_{r s}(i)\right) / t_{r s} q_{r s}\right)}{n(n-1)}
$$

where $\mathrm{BC}(i)$ is the betweenness centrality of intersection $i$; $t_{r s}$ is the number of the shortest paths between intersections $r$ and $s$, and $t_{r s}(i)$ is the number of the shortest paths between intersections $r$ and $s$ that go through the intersection $i ; q_{r s}$ is the traffic flow of the shortest paths between intersections $r$ and $s$, and $q_{r s}(i)$ is the traffic flow of the shortest paths between intersections $r$ and $s$ that go through the intersection $i$; and $n$ is the number of the intersections, and the formulation of $n(n-1) / 2$ is employed to normalize the betweenness centrality. The greater BC $(i)$ indicates that the intersection $i$ is more important.

The degree centrality indicates the property that an intersection connects with other intersections directly, which can be defined as

$$
\operatorname{DC}(i)=\frac{\sum_{j=1}^{n} a_{i j} a_{i j}}{(n-1) Q},
$$

where $a_{i j}$ is the adjacency factor and $Q$ is the total traffic flow on the roadway network. When there is a roadway segment between intersections $i$ and $j, a_{i j}=1$; otherwise, $a_{i j}=0$. 
Compared with the conventional CC (i), BC (i), and DC $(i)$ that used for node importance computing, the proposed CC (i), BC (i), and DC (i) account for the impacts of traffic flow, which could better represent the spatial topological relationship of roadway networks.

\subsection{Indicators of Intersection Traffic Safety. Regarding the} assessment of intersection traffic safety, the commonly employed methods could be classified into two categories [23]. One is a reactive approach, and the crash data that include historical crash records and predicted crashes could be used. Another is a proactive approach, and the Surrogate Measures of Safety (SMoS) were used to identify the crashprone locations where an observable noncrash event could lead to a crash $[24,25]$. Although the reactive approaches have many limitations such as missing crash records, small sample size, and unobserved causal factors of the crashes, the methods are essential for the greatest benefit of BSID because the observed and predicted crash counts indicate the effects of risk factors that are involving geometric design features, traffic conditions, and environmental characteristics. The SMoS approach, on the other hand, without relying on a huge number of crash data, developed the trajectorybased measures to identify the noncrash event that could be further converted into the corresponding crash frequency. In the research, the reactive and proactive approaches have been developed simultaneously since the traffic safety of intersection includes the interactions among human factors, vehicle characteristics, roadway design features, and traffic flow movement.

For the reactive approaches, the empirical Bayesian (EB) techniques that combine the benefit of observed and estimated crash frequencies have been employed [19]. The observed and estimated crash frequencies are weighted in a statistical model:

$$
Y_{i}=\left(1-\omega_{i}\right) y_{i o}+\omega_{i} y_{i p},
$$

where $Y_{i}$ is the expected crash frequency for intersection $i ; y_{i o}$ and $y_{i p}$ are the observed and estimated crash frequencies for intersection $i$ in the research period; and the weight factor $\omega_{i}$ represents the reliability level of the estimated crashes $y_{i p}$.

The estimated crash frequencies $y_{i p}$ could be computed by a negative binomial regression model:

$$
y_{i p}=\exp \left(\alpha+\sum_{j} \beta_{j} x_{i j}\right),
$$

where $\alpha$ is the intercept and $\beta_{j}$ is the regression coefficients of the corresponding explanatory variables $x_{i j}$.

Since not all the impact factors that could potentially cause the occurrences of traffic crashes could be observed and measured, there is a need to develop some effective and efficiency SMoS for intersection traffic safety screening. Commonly used SMoS, such as time-to-collision (TTC) [26] and postencroachment time (PET) [23], are inappropriate for intersection traffic safety screening since the characteristics of vehicle movements at intersections, such as frequently acceleration and nonlane-based vehicle movements, have not been taken into account. Based on Newton's equations of motion, a traffic crash could be occurred when the distances traveled by the following vehicle is equal to or greater than the sum of the initial relative distance between the leading and following vehicles and the distance traveled by the leading vehicle [27]:

$$
v_{f} t+\frac{1}{2} a_{f} t^{2} \geq v_{l} t+\frac{1}{2} a_{l} t^{2}+s
$$

where $v_{f}$ and $v_{l}$ represent the speed of the following and leading vehicles, respectively, $t$ is the time gap; $a_{f}$ and $a_{l}$ represent the acceleration of the following and leading vehicles, respectively, and $s$ denotes the initial relative distance.

Let $\Delta v$ and $\Delta a$ be the relative speed and acceleration of the interacting vehicles, respectively, the Modified TTC (MTTC) can be obtained from equation (5):

$$
\text { MMTC }= \begin{cases}\min \left(t_{1}, t_{2}\right), & \text { if } \Delta a, t_{1}, t_{2}>0, \\ t_{1}, & \text { if } \Delta a, t_{1}>0, t_{2} \leq 0, \\ t_{2}, & \text { if } \Delta a, t_{2}>0, t_{1} \leq 0, \\ t_{3}, & \text { if }\left(\Delta a \leq 0 \text { or } t_{1}, t_{2} \geq 0\right) \text { and } t_{3}>0,\end{cases}
$$

where $t_{1}$ and $t_{2}$ are the two values that are derived from equation (5); and $t_{3}$ is the original TTC:

$$
\begin{aligned}
& t_{1}=\frac{-\Delta v+\sqrt{\Delta v^{2}+2 \Delta a s}}{\Delta a}, \\
& t_{2}=\frac{-\Delta v-\sqrt{\Delta v^{2}+2 \Delta a s}}{\Delta a}, \\
& t_{3}=\frac{s}{\Delta v} .
\end{aligned}
$$

The MTTC could be computed based on the vehicle trajectories that are obtained from a video record. Other than the MTTC, to account for the effects of nonlane-based vehicle movements, a distance that is based on the longitudinal gap and lateral overlap is proposed to identify the vehicle interaction characteristics:

$$
d=\sqrt{\left(x_{l}-x_{f}-L_{l}\right)^{2}+\left(\left|y_{l}-y_{f}\right|-\frac{w_{l}}{2}-\frac{w_{f}}{2}\right)^{2}}
$$

where $\left(x_{l}, y_{l}\right)$ is the center coordinate of the leading vehicle, $\left(x_{f}, y_{f}\right)$ is the center coordinate of the following vehicle, $L_{l}$ is the length of the leading vehicle, $w_{l}$ is the width of the leading vehicle, and $w_{f}$ is the width of the following vehicle.

An interaction between the leading and following vehicles is identified as critical if the path of the two vehicles is overlapped. In other words, the interactions are critical if the $d$ is less than $\sqrt{L_{l}^{2}+1 / 2\left(w_{l}+w_{f}\right)^{2}}$. Such critical interactions could be identified for individual vehicle in the intersection area at every instant.

3.3. A Framework for Combining the Traffic Safety Assessment and Node Importance. To obtain more comprehensive and effective results, the multi-attribute decision-making 
(MADM) methods could be used to account for the effects of the proposed six indicators [28]. As the commonly used MADM methods, the Technique for Order Preference by Similarity to an Ideal Object (TOPSIS) methods and VIsekriterijumsko KOmpromisno Rangiranjie (VIKOR) methods have been proposed based on an aggregating function representing the concept of closeness to the ideal $[29,30]$. The TOPSIS methods compare the distance of individual alternative to the ideal and anti-ideal solution, and the VIKOR methods have been developed to provide compromise solutions to discrete multiple indicators that include noncommensurable and conflicting indicators. In the research, the VIKOR methods have been employed to screen and rank the intersection with multiple indicators since the indicators of traffic safety and node importance are not commensurable. In addition, a multilayer entropyweighed VIKOR (MEW-VIKOR) method is proposed to better account for the effects of traffic safety indicators and node importance indicators, simultaneously.

Suppose the set of indicators is $\mathbf{C}=\left\{c_{1}, c_{2}, \ldots, c_{m}\right\}$, then the normalized decision matrix can be expressed as

$$
\begin{aligned}
& \mathbf{D}=\left[\begin{array}{cccc}
x_{11} & x_{12} & \cdots & x_{1 m} \\
x_{21} & x_{22} & \cdots & x_{2 m} \\
\vdots & \vdots & \ddots & \vdots \\
x_{n 1} & x_{n 2} & \cdots & x_{n n}
\end{array}\right], \\
& x_{i j}=\frac{c_{i j}}{\sqrt{\sum_{i=1}^{n}\left(c_{i j}\right)^{2}}},
\end{aligned}
$$

where $x_{i j}$ is the normalized value of $c_{i j}$ and $c_{i j}$ is the value of $j_{\text {th }}$ indicator for the $i_{\text {th }}$ intersection, and $i=1,2, \ldots, n ; j=1,2$, ..., $m$.

To overcome the limitation of the conventional VIKOR methods that are using the subjective weigh factors, the entropy-weighted (EW) method is adopted to compute the weight of individual indicator. By accounting for the information entropy, the weight of the $j_{\text {th }}$ indicator of the proposed entropy-weighted VIKOR (EW-VIKOR) can be computed as

$$
w_{j}=\frac{1+(1 / \ln n) \sum_{i}^{n} e_{i j}}{\sum_{j=1}^{3}\left(1+(1 / \ln n) \sum_{i}^{n} e_{i j}\right)},
$$

where $e_{i j}$ is the information entropy of $j_{\text {th }}$ indicator for the $i_{\text {th }}$ intersection; $n$ is the number of analyzed intersections.

The $e_{i j}$ can be computed as

$$
\begin{aligned}
& e_{i j}=\frac{x_{i j}}{X_{j}} \ln \left(\frac{x_{i j}}{X_{j}}\right), \\
& X_{j}=\sum_{i=1}^{n} x_{i j} .
\end{aligned}
$$

The utility and regret measures for all intersections can be computed as

$$
\begin{aligned}
U_{i} & =\sum_{j=1}^{m} w_{j} \frac{\left(x_{j}^{*}-x_{i j}\right)}{\left(x_{j}^{*}-x_{j}^{-}\right)}, \\
R_{i} & =\max _{j}\left[w_{j}\left(x_{j}^{*}-x_{i j}\right)\left(x_{j}^{*}-x_{j}^{-}\right)\right],
\end{aligned}
$$

where $U_{i}$ is the utility measure; $R_{i}$ is the regret measure; and $x_{j}^{*}$ is the ideal solution, $x_{j}^{*}=\max _{i} x_{i j} ; x_{j}^{-}$is the negative solution, and $x_{j}^{-}=\min _{i} x_{i j}$.

Let $U^{*}=\min _{i} U_{i}, U^{-}=\max _{i} U_{i}, R^{*}=\min _{i} R_{i}$, and $R^{-}=\max _{i} R_{i}$, the EW-VIKOR index of importance of the $i_{\text {th }}$ intersection can be computed as follows:

$$
P_{i}=1-\alpha \frac{\left(U_{i}-U^{*}\right)}{\left(U^{-}-U^{*}\right)}+(1-\alpha) \frac{\left(R_{i}-R^{*}\right)}{\left(R^{-}-R^{*}\right)},
$$

where $\alpha$ is the weight for the strategy of maximum group utility, whereas $1-\alpha$ is the weight of the individual regret, here $\alpha=0.5$.

The greater the $P_{i}$ is, the more important the analyzed intersection is. The analyzed intersections can be sorted by the value of $P_{i}$. If $P_{i}=0$, the intersection is the least important and some maintenance methods should be taken into account to ensure that the interaction condition stays at a certain level. If $P_{i}=1$, the intersection is the most important and more resources of traffic safety improvements should be assigned and implemented.

In the research, to fully address the issues of combining the indicators of traffic safety assessment and node importance, four strategies have been proposed under the framework of intersection traffic safety screening and the MEW-VIKOR methods are developed, as shown in Figure 1:

(1) Strategy 1 is using the EW-VIKOR method to compute the ranking results based on the indicators of traffic safety assessment. The results of traffic safety assessment will be incorporated into the process of computing the node importance as an impact factor, and the EW-VIKOR method will be running for one more time.

(2) Strategy 2 is using the EW-VIKOR method to compute the ranking results based on the indicators of node importance. The results of node importance will be incorporated into the process of traffic safety assessment as an impact factor and the EW-VIKOR method will be running for one more time.

(3) Strategy 3 treated all the traffic safety assessment and node importance indicators equally, and the EWVIKOR will be used to compute the ranking results based on all the indicator set of traffic safety assessment and node importance.

(4) Strategy 4 is a MEW-VIKOR method. The first layer computes the ranking results based on the indicators of traffic safety assessment and node importance, respectively. The second layer computes the ranking results based on the results of first layer, which includes the ranking results of traffic safety assessment and node importance. The third layer is the final ranking results, which comprehensively account for 


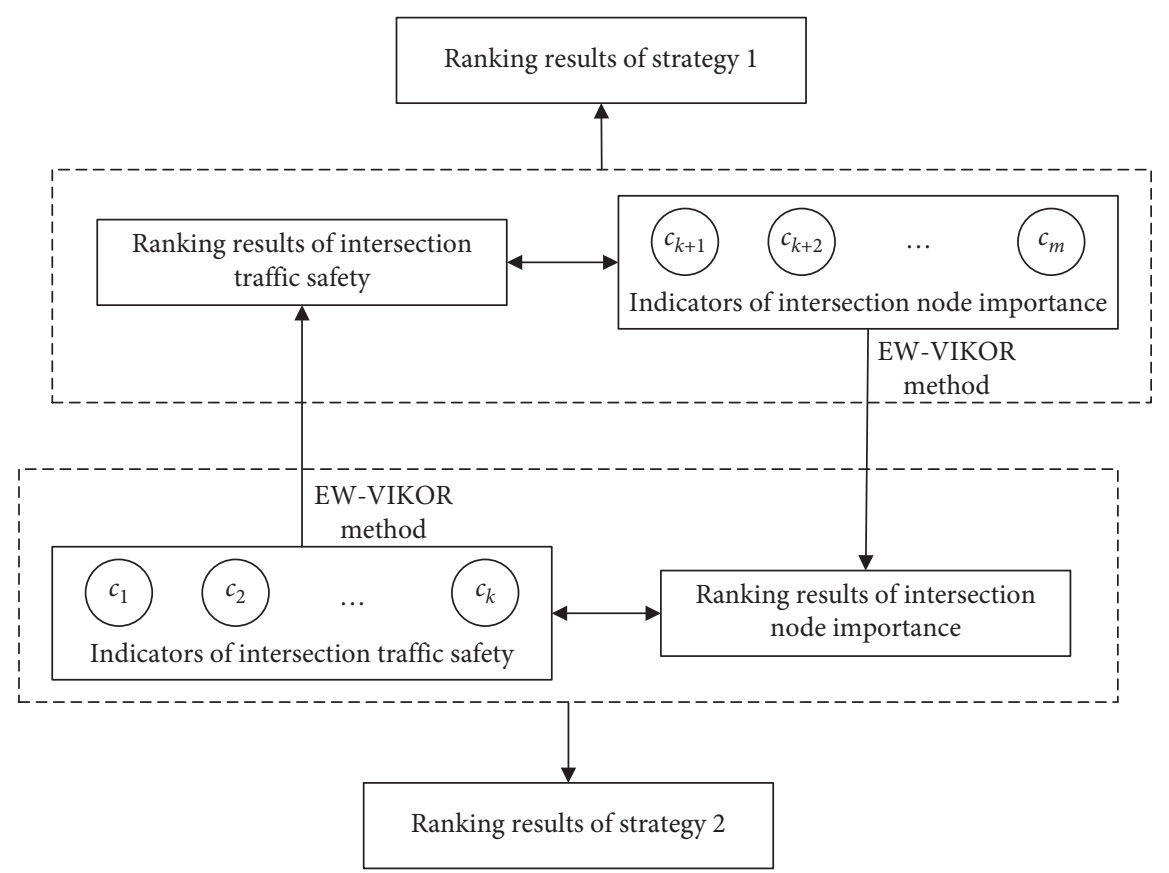

(a)

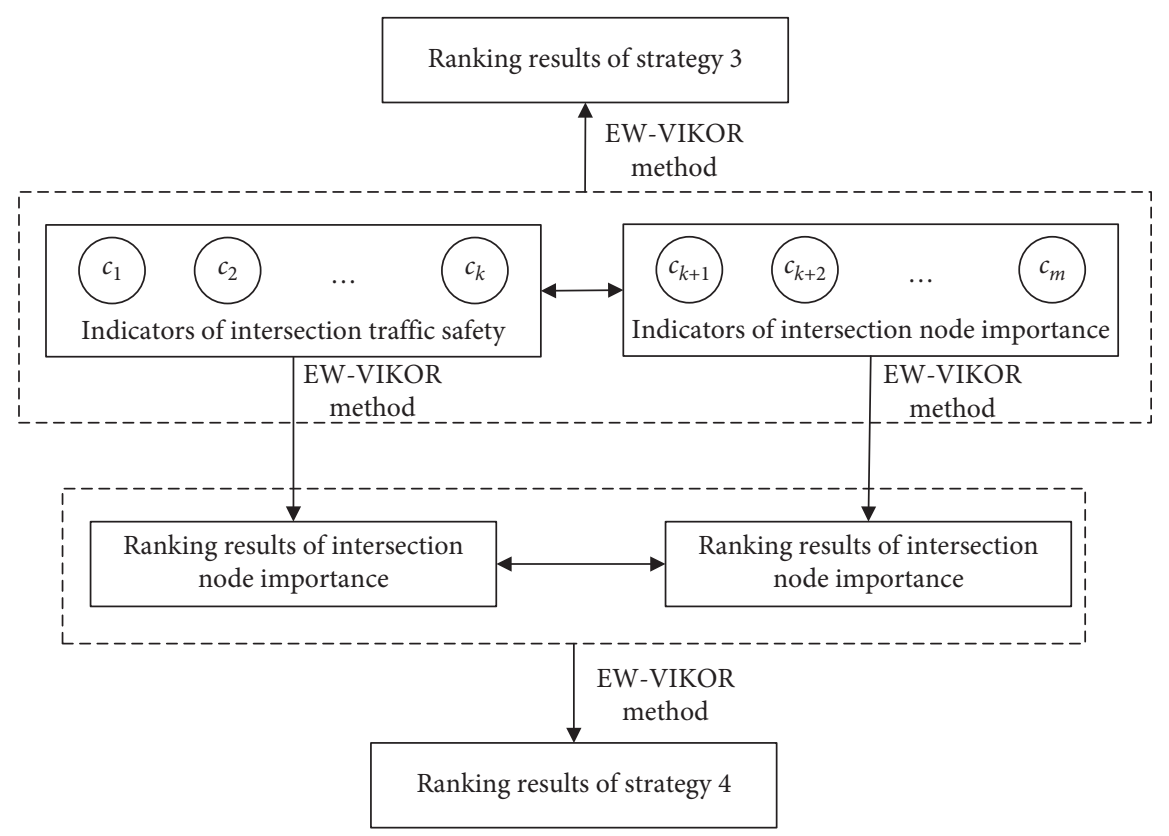

(b)

FIgURE 1: Flow chart of the proposed framework with four strategies: (a) strategies 1 and 2 and (b) strategies 3 and 4.

the effects of traffic safety assessment and node importance.

\section{Data}

In the research, six indicators have been developed from the perspectives of node importance and traffic safety, which demonstrate the complex interactions among the human factors, vehicle characteristics, roadway design features, and traffic flow movement. Based on the developed indicators, a framework with four strategies has been proposed for intersection traffic safety screening. To examine the effectiveness of the proposed indicators and verify the efficiency of the proposed framework with four strategies, a roadway network of an old town in Futian District, Shenzhen City, China, has been adopted, which includes 26 intersections and 52 roadway segments, as shown in Figure 2(a).

To compute the indicators of node importance, the topology of the roadway network needs to be extracted. The intersections are treated as the network nodes, and the roadway segment between the intersections are treated as the network edges. The topology of the roadway network of an 


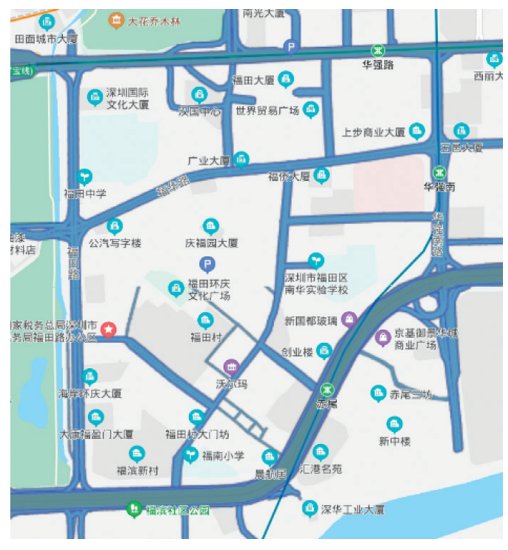

(a)

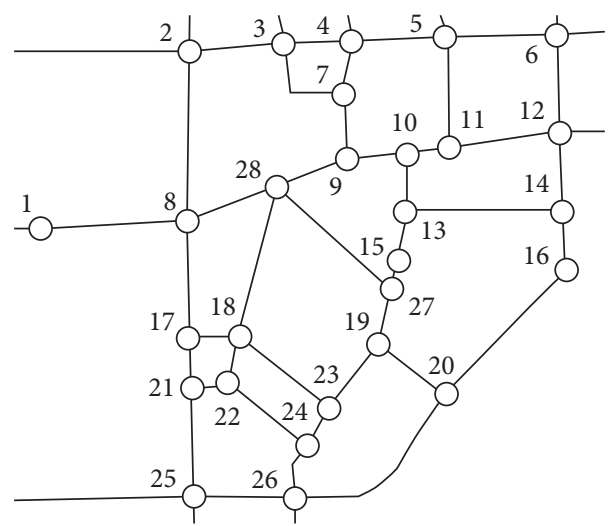

(b)

Figure 2: Roadway network of an old town in Futian District, Shenzhen City, China: (a) a map of research zone and (b) the topology of roadway network.

old town in Futian District is shown in Figure 2(b). The traffic flow of an individual segment in the roadway network can be considered as the weighting factors. The lengths of the roadway segments are measured to compute the shortest path between intersections $i$ and $j$. The Dijkstra algorithm is employed to compute the shortest path between $i$ and $j$. The statistics of all the shortest paths from $i$ to $j(j \neq i)$ are shown in Table 1.

Other than the data of segment length and the length of the shortest path, the information of the traffic flow is needed to compute the indicators of the node importance. In the research, instead of the annual average daily traffic (AADT), the capacity is used to measure the traffic flow of the shortest path because the AADT data are not available. The capacity of the individual segment is computed as the product of the number of through lanes and the capacity of the through lane. The capacity of the through lane is equal to 1700 pcu per lane. The statistics of the traffic flow of the shortest path are shown in Table 1 . The range of the traffic flow of the shortest path is from 13,852 to 37,526.

Regarding the indicators of traffic safety, to obtain $Y_{i}$, the $y_{i p}$ in equation (4) is computed by using the negative binomial (NB) regression model. The factors of speed limit, percent of trucks, and important measures of roadway design features, such as lane widths, median types, and shoulder widths are employed as the explanatory variables since they have shown significant impacts on the crash frequencies [31]. The study period of $y_{i p}$ and $y_{i o}$ is 5 years, and the weight factor $\omega_{i}$ is equal to 0.5 . The indicators of MMTC ( $i$ ) and $d_{i}$ are obtained from the video record with the recoding time of $30 \mathrm{~min}$. The calculation results are shown is Table 2 .

\section{Modeling Results}

Based on the indicators of traffic safety and node importance, the ranking results could be obtained by using the proposed MEW-VIKOR method. Using the three indicators of traffic safety, $Y_{i}$, MMTC (i), and $d_{i}$, the intersection ranking results have been obtained with the focus of conventional traffic safety evaluation. Using the three indicators
TABLE 1: Statistics of the shortest path and traffic flow.

\begin{tabular}{|c|c|c|c|c|c|c|c|c|}
\hline \multirow[t]{2}{*}{ ID } & \multicolumn{4}{|c|}{$\begin{array}{l}\text { Length of the shortest } \\
\text { path }\end{array}$} & \multicolumn{4}{|c|}{ Traffic flow of the shortest path } \\
\hline & Min & $\operatorname{Max}$ & Mean & SD & Min & Max & Mean & SD \\
\hline 1 & 0.40 & 1.77 & & & & & & \\
\hline 2 & .27 & 1.64 & & & & & & 15752 \\
\hline 3 & & & & & & & & \\
\hline 4 & & & & & & & & \\
\hline 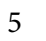 & & 1.6 & & & & & & \\
\hline 0 & & 1.9 & & & & & & \\
\hline & & & & & & & & \\
\hline & & & & & & & & \\
\hline & & & & & & & & \\
\hline 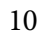 & & & & & & & & \\
\hline & & & & & & & & \\
\hline & & & & & & & & \\
\hline & & & & & & & & \\
\hline & & & & & & & & \\
\hline & & & & & & & & \\
\hline 1 & & & & & & & & \\
\hline & & & & & & & & \\
\hline & & 1.4 & & & & & & 539 \\
\hline & & & & & & & & \\
\hline 20 & 0.21 & 1.5 & & & & & & \\
\hline 21 & & & & & & & & 12082 \\
\hline 22 & & & & & & & & \\
\hline 23 & & & & & & & & \\
\hline 24 & & 1.5 & & & & & & 1217 \\
\hline 25 & & 1.9 & & & & & & \\
\hline 26 & & 1.6 & & & & & & 13280 \\
\hline 27 & & & & & & & & \\
\hline & 0.21 & 1.12 & 0.65 & 0.24 & 3400 & 37400 & 13852 & 8271 \\
\hline
\end{tabular}

of node importance, CC (i), BC (i), and DC (i), the intersection ranking results with the focus of roadway network function and traffic flow have been obtained. Using the six indicators and the proposed framework, the intersection ranking results have been obtained with four strategies. The intersection ranking results of the proposed approaches with the different focuses are shown in Table 3. The comparison results are shown in Figure 3. 
TABLE 2: Indicators of intersection node importance and traffic safety.

\begin{tabular}{|c|c|c|c|c|c|c|}
\hline \multirow{2}{*}{ ID } & \multicolumn{3}{|c|}{ Indicators of node importance } & \multicolumn{3}{|c|}{ Indicators of traffic safety } \\
\hline & $\mathrm{CC}(i)$ & $\mathrm{BC}(i)$ & $\mathrm{DC}(i)$ & $Y_{i}$ & $\operatorname{MMTC}(i)$ & $d_{i}$ \\
\hline 1 & 0.88 & 0.00 & 0.04 & 6 & 0.32 & 6.84 \\
\hline 2 & 0.99 & 0.03 & 0.07 & 6 & 0.60 & 6.77 \\
\hline 3 & 1.05 & 0.06 & 0.11 & 7 & 1.14 & 6.42 \\
\hline 4 & 1.18 & 0.05 & 0.11 & 8 & 1.24 & 6.18 \\
\hline 5 & 1.13 & 0.06 & 0.11 & 4 & 0.74 & 6.26 \\
\hline 6 & 0.93 & 0.04 & 0.07 & 0 & 0.45 & 6.18 \\
\hline 7 & 1.32 & 0.21 & 0.11 & 7 & 0.52 & 6.96 \\
\hline 8 & 1.33 & 0.22 & 0.15 & 5 & 1.22 & 7.05 \\
\hline 9 & 1.55 & 0.53 & 0.11 & 0 & 0.35 & 6.20 \\
\hline 10 & 1.59 & 0.63 & 0.11 & 0 & 0.66 & 6.67 \\
\hline 11 & 1.43 & 0.28 & 0.11 & 0 & 0.63 & 6.90 \\
\hline 12 & 1.11 & 0.14 & 0.11 & 0 & 0.80 & 6.28 \\
\hline 13 & 1.53 & 0.50 & 0.11 & 5 & 1.03 & 6.09 \\
\hline 14 & 1.07 & 0.10 & 0.11 & 0 & 1.05 & 6.67 \\
\hline 15 & 1.52 & 0.41 & 0.07 & 0 & 0.56 & 6.83 \\
\hline 16 & 1.00 & 0.03 & 0.07 & 0 & 0.65 & 6.38 \\
\hline 17 & 1.22 & 0.12 & 0.11 & 8 & 1.12 & 6.34 \\
\hline 18 & 1.34 & 0.24 & 0.15 & 0 & 0.26 & 7.02 \\
\hline 19 & 1.46 & 0.47 & 0.11 & 7 & 0.70 & 6.47 \\
\hline 20 & 1.20 & 0.06 & 0.11 & 0 & 0.61 & 6.67 \\
\hline 21 & 1.15 & 0.12 & 0.11 & 6 & 0.36 & 6.49 \\
\hline 22 & 1.23 & 0.20 & 0.11 & 7 & 0.62 & 6.62 \\
\hline 23 & 1.34 & 0.35 & 0.11 & 5 & 0.50 & 6.40 \\
\hline 24 & 1.23 & 0.25 & 0.11 & 7 & 1.05 & 6.40 \\
\hline 25 & 0.98 & 0.00 & 0.07 & 0 & 0.41 & 6.36 \\
\hline 26 & 1.08 & 0.07 & 0.11 & 5 & 0.35 & 6.15 \\
\hline 27 & 1.53 & 0.46 & 0.11 & 0 & 0.35 & 6.50 \\
\hline 28 & 1.54 & 0.37 & 0.15 & 8 & 1.09 & 6.21 \\
\hline
\end{tabular}

TABLE 3: The results of intersection ranking.

\begin{tabular}{|c|c|c|c|c|c|c|}
\hline Intersection ID & Results of traffic safety & Results of node importance & Strategy 1 & Strategy 2 & Strategy 3 & Strategy 4 \\
\hline 1 & 0.20 & 0.00 & 0.00 & 0.15 & 0.00 & 0.00 \\
\hline 2 & 0.44 & 0.14 & 0.62 & 0.28 & 0.56 & 0.21 \\
\hline 3 & 0.94 & 0.23 & 0.79 & 0.39 & 0.61 & 0.46 \\
\hline 4 & 1.00 & 0.36 & 0.87 & 0.56 & 0.65 & 0.60 \\
\hline 5 & 0.51 & 0.32 & 0.70 & 0.41 & 0.64 & 0.40 \\
\hline 6 & 0.15 & 0.08 & 0.51 & 0.05 & 0.29 & 0.05 \\
\hline 7 & 0.40 & 0.54 & 0.76 & 0.74 & 0.76 & 0.52 \\
\hline 8 & 0.92 & 0.57 & 0.91 & 0.72 & 0.77 & 0.78 \\
\hline 9 & 0.06 & 0.92 & 0.75 & 0.64 & 0.95 & 0.33 \\
\hline 10 & 0.36 & 1.00 & 0.84 & 0.68 & 1.00 & 0.64 \\
\hline 11 & 0.34 & 0.66 & 0.79 & 0.54 & 0.83 & 0.50 \\
\hline 12 & 0.45 & 0.32 & 0.67 & 0.31 & 0.65 & 0.39 \\
\hline 13 & 0.79 & 0.88 & 0.95 & 0.95 & 0.94 & 0.99 \\
\hline 14 & 0.56 & 0.26 & 0.69 & 0.25 & 0.62 & 0.36 \\
\hline 15 & 0.27 & 0.79 & 0.81 & 0.59 & 0.90 & 0.49 \\
\hline 16 & 0.34 & 0.15 & 0.60 & 0.13 & 0.56 & 0.18 \\
\hline 17 & 0.95 & 0.43 & 0.87 & 0.65 & 0.69 & 0.65 \\
\hline 18 & 0.00 & 0.59 & 0.66 & 0.51 & 0.79 & 0.18 \\
\hline 19 & 0.55 & 0.82 & 0.85 & 0.99 & 0.90 & 0.75 \\
\hline 20 & 0.31 & 0.37 & 0.69 & 0.37 & 0.66 & 0.38 \\
\hline 21 & 0.20 & 0.35 & 0.62 & 0.50 & 0.66 & 0.27 \\
\hline 22 & 0.48 & 0.47 & 0.74 & 0.66 & 0.72 & 0.54 \\
\hline 23 & 0.30 & 0.64 & 0.73 & 0.78 & 0.81 & 0.46 \\
\hline 24 & 0.87 & 0.49 & 0.84 & 0.69 & 0.74 & 0.68 \\
\hline 25 & 0.11 & 0.11 & 0.52 & 0.09 & 0.52 & 0.07 \\
\hline 26 & 0.16 & 0.26 & 0.58 & 0.37 & 0.62 & 0.20 \\
\hline 27 & 0.07 & 0.84 & 0.75 & 0.61 & 0.92 & 0.32 \\
\hline 28 & 0.92 & 0.78 & 1.00 & 1.00 & 0.91 & 0.99 \\
\hline
\end{tabular}




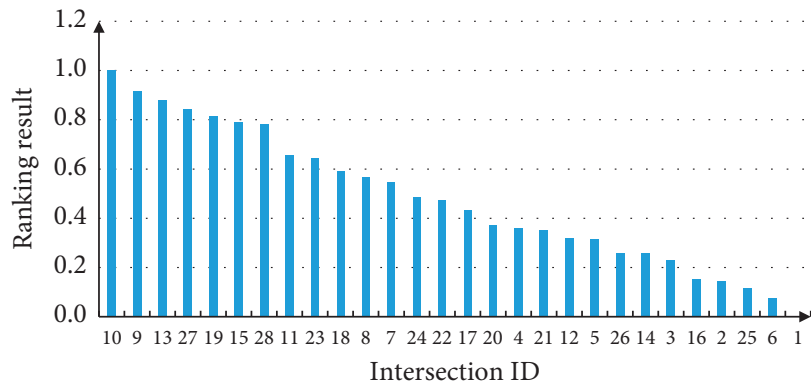

(a)

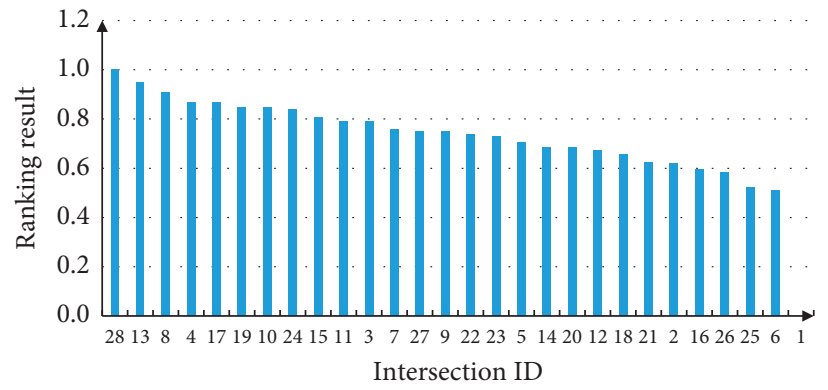

(c)

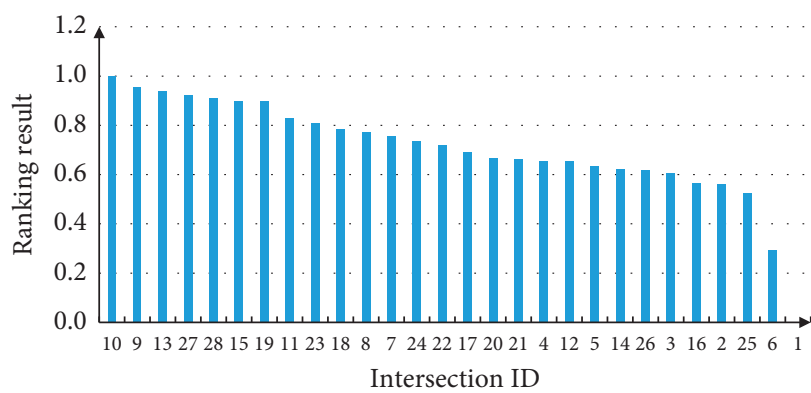

(e)

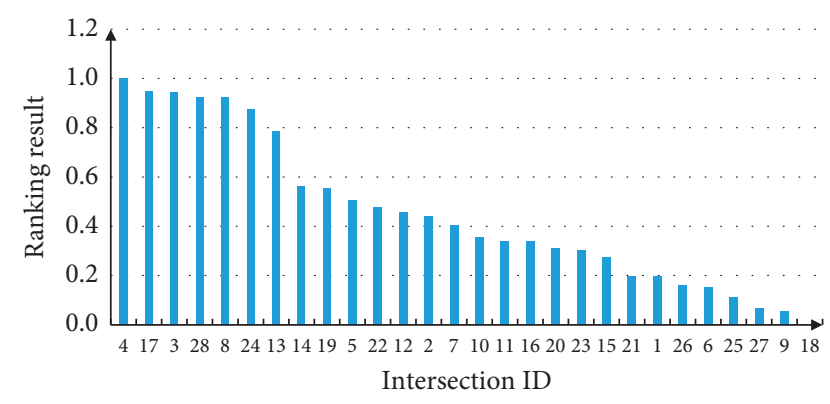

(b)

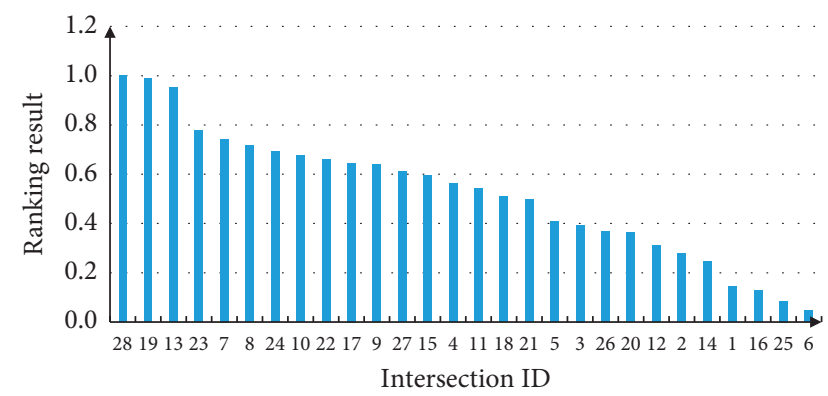

(d)

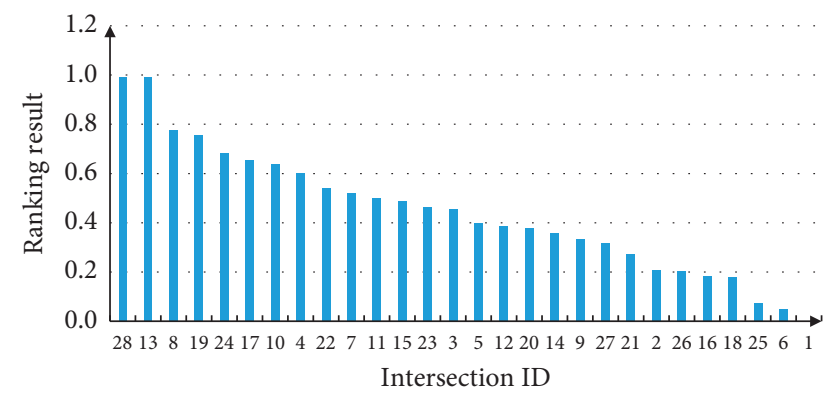

(f)

FiguRE 3: Comparison of the ranking results with analyzed intersections: (a) ranking results of node importance, (b) ranking results of traffic safety, (c) ranking results of strategy 1, (d) ranking results of strategy 2, (e) ranking results of strategy 3, and (f) ranking results of strategy 4.

From the comparison, the results indicate that strategies 1 and 3 have the similar pattern and changing trend, which is different compared to the results of node importance and traffic safety. The strategies 2 and 4 have the similar pattern and changing trend, which is a compromise solution of the node importance and traffic safety. In other words, strategies 2 and 4 represent the ranking result features of node importance and traffic safety simultaneously.

To further verify the performance of the proposed methods, the consistency test has been applied. With the ranking results of two methods, the consistency test analyzed consistency rate, which is a ratio of the intersections with same ranking order in total intersections, and the results are shown in Figure 4. The results demonstrated that the consistency rate between the ranking results of strategy 3 and node importance is $78.57 \%$, which is significantly greater than the others. The ranking results of strategy 3 represent more roadway network function and traffic flow characteristics compared with other strategies.
The consistency rate between the ranking results of traffic safety and strategies 1,2 , and 3 is 0 . The consistency rate between the ranking results of traffic safety and strategy 4 is $7.14 \%$. The findings indicate that ranking results of strategy 4 represent more traffic safety assessment features compared with the other strategies. In addition, the consistency rate between the ranking results of strategy 4 and the node importance is $14.29 \%$, which is greater than the consistency rate between strategy 4 and the traffic safety. Though strategy 4 represents more node importance features compared with the traffic safety, strategy 4 represents the superior balance between roadway network function and traffic safety assessment.

The results of the consistency test between the four strategies are shown in Figure 4(b). The results show that strategies 1 and 4 have the greatest similarity, and the consistency rate of the ranking results is $32.14 \%$. The consistency rate between the strategies 1 and 3 is $17.86 \%$, and between the strategies 3 and 4 is $14.29 \%$. The consistency rate of the rest of strategy combinations is $7.14 \%$. The findings 


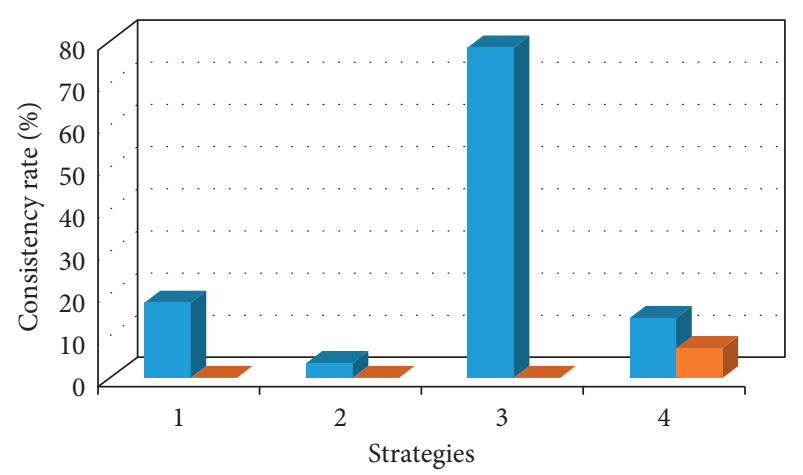

- Compared to the node importance

- Compared to the traffic safety

(a)

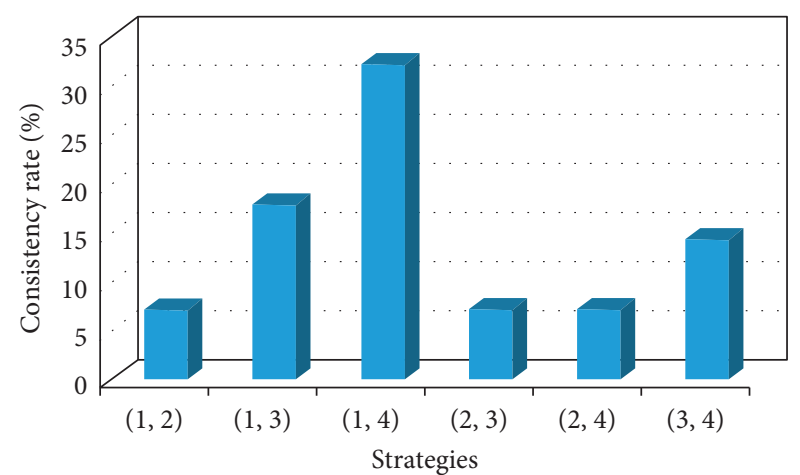

(b)

FIGURE 4: Consistency test results of the proposed methods: (a) the proposed four strategies compared the node importance and traffic safety and (b) comparison between the four strategies.

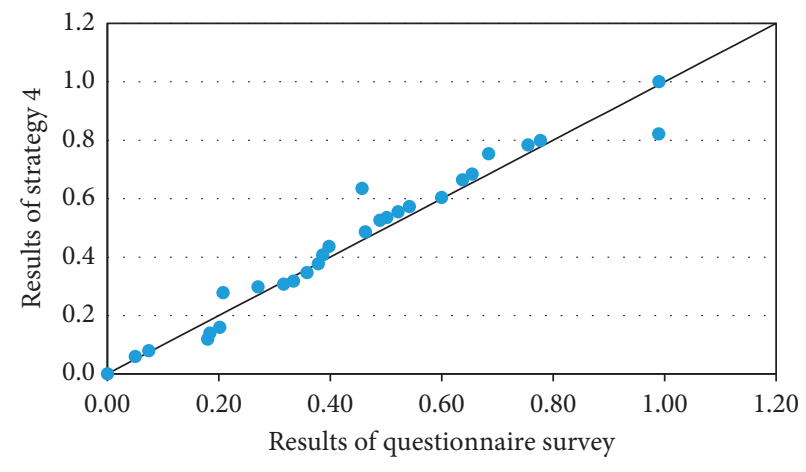

(a)

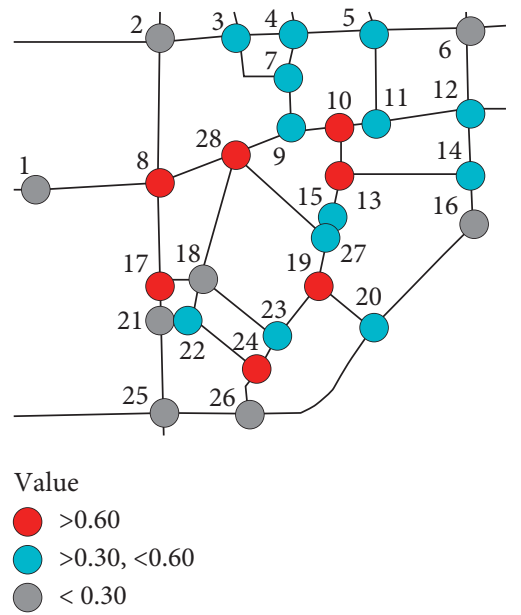

(b)

Figure 5: Comparison between the results of questionnaire survey and the proposed framework with strategy 4: (a) compared to the questionnaire survey and (b) differences with ranking value.

indicate that the strategies 1 and 4 could provide consistency results that cover both perspectives of traffic safety and node importance. Combined the comparison results and the consistency test, the proposed framework with strategy 4 is recommended for intersection traffic safety screening, since it has both merits of node importance and traffic safety.

To verify the effectiveness of the proposed method with strategy 4 , a questionnaire survey has been conducted. In total, 42 people who are from the government office, research institute, and public have participated the survey. Each participant ranked the 28 intersections according to their own expertise and experience. The ranking results of the poll have been analyzed and compared with the proposed framework with strategy 4, which are shown in Figure 5(a). The results of the proposed framework with strategy 4 are closely approximate to the results of the questionnaire survey. The consistency rate between the survey results and the proposed framework with strategy 4 is 85.71 . The findings indicate the ranking results could represent the attitude and expectation of the public and agencies.

The proposed framework could be used as the method of BSID with an appropriate threshold setting. The ranking results with the values that are greater than the threshold could be the intersections that are the black spots. In the research, for the proposed framework with strategy 4 , when the threshold is setting to 0.60 , the intersection of $8,10,13$, $17,19,24$, and 28 have been identified as the black spots, as shown in Figure 5(b). The results are consistent with the intersection safety investigation, and the rate of the falsepositive cases and false-negative cases is 0 . The false-positive case is an intersection involved in safety investigation while it is not needed, and a false-negative case is not involving a site in safety investigation while it is needed. The rates of false-positive and false-negative cases in identifying black spots are the assessing standards in justifying the performance of the traffic safety screening method. The findings 
show that the proposed framework with strategy 4 contributes significantly to the reduction of false-positive and false-negative cases in identifying the black spot intersections.

\section{Conclusions}

In the research, a framework with the indicators that are from the perspectives of complex network and traffic safety is proposed for the intersection traffic safety screening. By incorporating the traffic flow and the characteristics of roadway network topology, three indicators have been developed to measure the node importance. The observed and estimated crash frequencies, modified time-to-collision, and a distance that describes the nonlane-based movements at intersection have been proposed from the perspective of traffic safety assessment. A MEW-VIKOR approach is proposed to compute the ranking results, and four strategies have been developed to account for the effects of the six indicators simultaneously. To verify the effectiveness of the proposed framework for intersection traffic safety screening, a roadway network with 28 intersections in Shenzhen has been adopted.

The ranking results of the proposed framework with two layers could represent the features of traffic safety and the characteristics of node importance and satisfy the expectation from the public, government, and research institutes. With an appropriate threshold setting, the ranking results are consistent with the intersection safety investigation and contribute significantly to the reduction of false-positive and false-negative cases in identifying the black spot intersections.

The inappropriate designs at intersection can cause safety problems and impact the operation efficiency of neighboring zones and even the overall network. The screening, ranking, and identification of black spots are the first steps of the traffic safety management process. Errors and bias in black spot identification might result in the inefficient use of resources for traffic safety improvements and impact the effectiveness of the traffic safety management. The proposed framework could be used as a guideline to develop traffic management policies, enhance the level of traffic management, and reduce the impacts of traffic crashes and congestion.

\section{Data Availability}

The data will be available upon request to the corresponding author.

\section{Conflicts of Interest}

The authors declare that there are no conflicts of interest.

\section{Acknowledgments}

This research was supported by funding provided by the National Key R\&D Program of China (Grant number: 2019YFF0301400).

\section{References}

[1] C. Dong, D. B. Clarke, S. H. Richards, and B. Huang, "Differences in passenger car and large truck involved crash frequencies at urban signalized intersections: an exploratory analysis," Accident Analysis \& Prevention, vol. 62, pp. 87-94, 2014.

[2] C. Dong, S. H. Richards, D. B. Clarke, X. Zhou, and Z. Ma, "Examining signalized intersection crash frequency using multivariate zero-inflated Poisson regression," Safety Science, vol. 70, pp. 63-69, 2014.

[3] N. Levine, "Houston, Texas, metropolitan traffic safety planning program," Transportation Research Record: Journal of the Transportation Research Board, vol. 1969, no. 1, pp. 92-100, 2006.

[4] X. Qin, M. A. Sultana, M. V. Chitturi, and D. A. Noyce, "Developing truck corridor crash severity index," Transportation Research Record: Journal of the Transportation Research Board, vol. 2386, no. 1, pp. 103-111, 2013.

[5] ITT Corporation, Study Analyst User's Manual, Colorado Springs, CO, USA, 2011.

[6] M. H. Rahman, M. Abdel-Aty, J. Lee, and M. S. Rahman, "Enhancing traffic safety at school zones by operation and engineering countermeasures: a microscopic simulation approach," Simulation Modelling Practice and Theory, vol. 94, pp. 334-348, 2019.

[7] L. B. Meuleners, D. Hendrie, A. H. Lee, and M. Legge, "Effectiveness of the black spot programs in western Australia," Accident Analysis \& Prevention, vol. 40, no. 3, pp. 1211-1216, 2008.

[8] F. Yakar, "Identification of accident-prone road sections by using relative frequency method," PROMET-Traffic \& Transportation, vol. 27, no. 6, pp. 539-547, 2015.

[9] A. Borsos, S. Cafiso, C. D’Agostino, and D. Miletics, "Comparison of Italian and Hungarian black spot ranking," Transportation Research Procedia, vol. 14, pp. 2148-2157, 2016.

[10] W. Cheng and S. P. Washington, "Experimental evaluation of hotspot identification methods," Accident Analysis \& Prevention, vol. 37, no. 5, pp. 870-881, 2005.

[11] W. Cheng and S. Washington, "New criteria for evaluating methods of identifying hot spots," Transportation Research Record: Journal of the Transportation Research Board, vol. 2083, no. 1, pp. 76-85, 2008.

[12] E. De. Pauw, S. Daniels, T. Brijs, E. Hermans, and G. Wets, "Safety effects of an extensive black spot treatment programme in Flanders-Belgium," Accident Analysis and Prevention, vol. 66, pp. 72-79, 2014.

[13] N. Veeramisti, A. Paz, and J. Baker, "A framework for corridor-level traffic safety network screening and its implementation using Business Intelligence," Safety Science, vol. 121, pp. 100-110, 2020.

[14] A. Montella, "Safety reviews of existing roads," Transportation Research Record: Journal of the Transportation Research Board, vol. 1922, no. 1, pp. 62-72, 2005.

[15] Z. Fan, C. Liu, D. Cai, and S. Yue, "Research on black spot identification of safety in urban traffic accidents based on machine learning method," Safety Science, vol. 118, pp. 607616, 2019.

[16] S. Cafiso and G. Di Silvestro, "Performance of safety indicators in identification of black spots on two-lane rural roads," Transportation Research Record: Journal of the Transportation Research Board, vol. 2237, no. 1, pp. 78-87, 2011. 
[17] K. Geurts, G. Wets, T. Brijs, and K. Vanhoof, "Identification and ranking of black spots: sensitivity analysis," Transportation Research Record: Journal of the Transportation Research Board, vol. 1897, no. 1, pp. 34-42, 2004.

[18] A. Montella, "A comparative analysis of hotspot identification methods," Accident Analysis \& Prevention, vol. 42, no. 2, pp. 571-581, 2010.

[19] M. Ghadi and Áö Török, “A comparative analysis of black spot identification methods and road accident segmentation methods," Accident Analysis \& Prevention, vol. 128, pp. 1-7, 2019.

[20] Y. Yang, L. Yu, X. Wang et al., "A novel method to evaluate node importance in complex networks," Physica A: Statistical Mechanics and Its Applications, vol. 526, Article ID 121118, 2019.

[21] L. C. Freeman, "Centrality in social networks conceptual clarification," Social Networks, vol. 1, no. 3, pp. 215-239, 1978.

[22] M. E. J. Newman, "A measure of betweenness centrality based on random walks," Social Networks, vol. 27, no. 1, pp. 39-54, 2005.

[23] A. Charly and T. V. Mathew, "Estimation of traffic conflicts using precise lateral position and width of vehicles for safety assessment," Accident Analysis \& Prevention, vol. 132, Article ID 105264, 2019.

[24] J. Wu, H. Xu, Y. Zheng, and Z. Tian, "A novel method of vehicle-pedestrian near-crash identification with roadside LiDAR data," Accident Analysis \& Prevention, vol. 121, pp. 238-249, 2018.

[25] T. Fu, L. Miranda-moreno, and N. Saunier, "A novel framework to evaluate pedestrian safety at non-signalized locations," Accident Analysis \& Prevention, vol. 111, pp. 2333, 2018

[26] H. Behbahani, N. Nadimi, and S. S. Naseralavi, "New timebased surrogate safety measure to assess crash risk in carfollowing scenarios," Transportation Letters, vol. 7, no. 4, pp. 229-238, 2015.

[27] K. Ozbay, H. Yang, B. Bartin, and S. Mudigonda, "Derivation and validation of new simulation-based surrogate safety measure," Transportation Research Record: Journal of the Transportation Research Board, vol. 2083, no. 1, pp. 105-113, 2008.

[28] S. Opricovic and G.-H. Tzeng, "Compromise solution by MCDM methods: a comparative analysis of VIKOR and TOPSIS," European Journal of Operational Research, vol. 156, no. 2, pp. 445-455, 2004.

[29] Z. Liu, C. Jiang, J. Wang, and H. Yu, "The node importance in actual complex networks based on a multi-attribute ranking method," Knowledge-Based Systems, vol. 84, pp. 56-66, 2015.

[30] N. Ploskas and J. Papathanasiou, "A decision support system for multiple criteria alternative ranking using TOPSIS and VIKOR in fuzzy and nonfuzzy environments," Fuzzy Sets and Systems, vol. 377, pp. 1-30, 2019.

[31] C. Dong, C. Shao, D. B. Clarke, and S. S. Nambisan, "An innovative approach for traffic crash estimation and prediction on accommodating unobserved heterogeneities," Transportation Research Part B: Methodological, vol. 118, pp. 407-428, 2018. 